\title{
Reflets
}

Revue ontaroise d'intervention sociale et communautaire

\section{Profil épidémiologique des francophones de l'Ontario : les faits saillants revisités et comparés}

\section{Françoise Boudreau et Diane Farmer}

Volume 5, numéro 2, automne 1999

La santé des francophones de l’Ontario

URI : https://id.erudit.org/iderudit/026271ar

DOI : https://doi.org/10.7202/026271ar

Aller au sommaire du numéro

Éditeur(s)

Reflets : Revue ontaroise d'intervention sociale et communautaire

ISSN

1203-4576 (imprimé)

1712-8498 (numérique)

Découvrir la revue

Citer cet article

Boudreau, F. \& Farmer, D. (1999). Profil épidémiologique des francophones de l’Ontario : les faits saillants revisités et comparés. Reflets, 5(2), 103-108.

https://doi.org/10.7202/026271ar

Tous droits réservés (C) Reflets : Revue ontaroise d'intervention sociale et communautaire, 1999
Ce document est protégé par la loi sur le droit d'auteur. L'utilisation des services d’Érudit (y compris la reproduction) est assujettie à sa politique d'utilisation que vous pouvez consulter en ligne.

https://apropos.erudit.org/fr/usagers/politique-dutilisation/ 


\section{Profil épidémiologique des francophones de 1'Ontario: les faits saillants revisités et comparés}

\section{F rançoise $B$ oudrea ${ }^{1}$ et $D$ iane $F$ armer}

N ous présenterons, dans cet article, les faits saillants de l'Enquête sur la santé de l'O ntario de 1990. D éjà, cette enquête remonte à plusieurs années et les tendances observées doivent donc être comprises dans leur contexte, en plus de tenir compte des limites posées par l'enquête à l'époque. Les données de 1996 sont maintenant disponibles. $\mathrm{D}$ ans l'article de $\mathrm{H}$ ébert et Picard dans ce numéro de $R$ eflets, un profil nous est présenté, plus exhaustif et alimenté par de récentes études. Enrichis par cette nouvelle réflexion, il sera intéressant de voir dans quelle mesure certaines des tendances observées en 1990 peuvent apporter un éclairage aux phénomènes rapportés aujourd'hui.

L'étude, «Profil épidémiologique desfrancophones del'O ntario : les faits saillants revisités et comparés ", s'inscrit dans le cadre d'une recherche plus vaste sur la santé des femmes francophones, initiée par laTable féministe francophone de concertation provinciale de I'O ntario². L'étude épidémiologique visait à tracer, pour la première fois, le profil de santé des Franco-O ntariennes et des Franco0 ntariensà partir des données de l'Enquête sur la santé en 0 ntario de 1990 (ESO 1990) et à en dégager les problématiquesémergentes 3 . Compte tenu du contexte politique minoritaire de la francophonie ontarienne, les données ont été abordées dans une perspective comparative principalement entre les groupes «francophones » et « anglophones», sans toutefois négliger de souligner certains traits plus typiques de la population allophone. L'enquête permettait de 
poser un regard quantifié et préliminaire sur une réalité à la fois complexe et mouvante. La mise à jour de ces données nous invite à proposer une réflexion qui permettra la mise en contexte des tendances observées et partira à la quête de sens.

\section{Quelques faits à souligner}

"L'évaluation que fait une personne de sa santé a un effet déterminant sur son mieux-être »

«Par exemple, vingtdeux pour cent $(22 \%)$ des hommes anglophones entre 45 et 64 ans se disent en excellente santé comparativement à $15 \%$ des hommes francophones, »
En général, lesfrancophoneset lesanglophones présentent desprofils de santéqui paraissent assez semblables surtout lorsqu'on les compare à la population allophone L'âge, le sexe et le statut socio-économique demeurent de grands facteurs qui influencent l'état de santé d'une personne. Cependant, un regard plus fin présente des différences notables entre ces populations, lai ssant ainsi présager que l'ethnicité intervient aussi au niveau de la santé des francophones.

$D$ 'abord, un découpage par tranche d'âge révèle que les francophones de 45 ans et plus, dont lesaînés, présentent davantage de problèmes de santé. Par exemple, plus d'aînés anglophones que francophones disent ne présenter aucun problème de santé, tant chez les hommes ( $15 \%$ vs $9 \%$ ) que chez lesfemmes ( $12 \%$ vs $7 \%$ ).

En fait de types de problèmes de santéencourus, lesfrancophones des deux sexes présentent proportionnellement plus de problèmes musculaires-squelettiques que les anglophones, héritage possible d'une tradition plus ouvrière chez les Franco- 0 ntariens. A joutons que les hommes francophones de 65 ans et plus rapportent deux fois plus d'accidents que leur contrepartie anglophone.

L'évaluation que fait une personne de sa santé a un effet déterminant sur son mieux-être. C ette «construction » de la réalité est influencée par les attentes personnelles, les expériences passées avec la maladie, les références culturelles et les modèles de santé présents dansl'environnement. À nouveau, on note une différence importante entre les groupes linguistiques, notamment chez les adultes de 45 ans et plus. Par exemple, vingt- deux pour cent (22\%) deshommesanglophonesentre 45 et 64 ans se disent en excellente santé comparativement à $15 \%$ des hommes francophones. 
C ependant, la variable la plus révélatrice du sentiment de bienêtre est les idéations suicidaires et la tentative de suicide. Ici, $13 \%$ des francophones et $11 \%$ des anglophones disent avoir envisagé le suicide à un moment donné de leur vie.

Le soutien social et le climat familial sont d'autres facteurs qui influencent l'état de santé d'une population. La majorité des 0 ntariennes et 0 ntariens, incluant les francophones, bénéficient d'un soutien social «moyen ». Soulignons que les francophones de 45 à 64 ans disent bénéficier d'un soutien social «élevé » dans une plus grande proportion. Par ailleurs, le climat familial varie considérablement en fonction de l'âge et du sexe et à ce chapitre, les femmes francophones connaissent les changements les plus radicaux. En effet, la proportion de femmes qui disent vivre dans un climat familial harmonieux, passe de $80 \%$ pour la cohorte des 20 à 44 ansà moins de $60 \%$ chez les 45 à 64 ans. 0 n observe la même tendance chez les femmes anglophones, bien que l'écart entre les deux cohortes est largement inférieure. Du côté des aînés, les francophones semblent moins nombreux à bénéficier d'un climat familial harmonieux. Et encore là, les femmes sont plus susceptibles d'éprouver son absence.

L'analyse du profil de santé de la population francophone de I'O ntario tient aussi compte du processus de maintien de la santé, en étudiant les moyens utilisés pour demeurer en santé tels que la nutrition, les risques compromettant la santé et l'usage des services de santé. Les références culturelles, tout comme le style de vie et le statut socio-économique, viennent à nouveau influencer les choix

«... l'enquête a révélé qu'on trouve, chez les francophones, $7 \%$ plus de fumeurs que chez les anglophones $L$ es francophones ont aussi tendance à consommer davantage de médicaments sur ordonnance » posés pour demeurer en santé. Par exemple, l'enquête arévélé qu'on trouve, chez les francophones, $7 \%$ plus de fumeurs que chez les anglophones. Les francophones ont aussi tendance à consommer davantage de médicaments sur ordonnance. $C$ 'est particulièrement le cas des aînés. Côté alimentation, les 0 ntariens, incluant les francophones, consomment plus de matières grasses que la norme canadienne recommandée. Les hommes francophones de 45 à 64 anssont ceux qui en consomment le plus. Fait inquiétant, $51 \%$ des adolescents francophones de 16 à 19 ans et $41 \%$ des adolescentes ont déclaré que leur routine professionnelle était « toujours dangereuse » comparativement au tiers des jeunes anglophones ${ }^{4}$. 
En ce qui a trait à l'utilisation des service de santé, plus de neuf O ntariens sur dix, incluant les francophones, font moins de 12 visites par année chez leur omnipraticien ou leur médecin spécialiste. En fait de type de professionnels consultés, les francophones ont moins tendance à consulter des spécialistes. Soulignons que la proportion de francophones est plus élevée parmi lespersonnesqui consultent fréquemment un professionnel de la santé (9 fois ou plus par année), surtout chez les 45 à 64 ans. Enfin, les francophones sont plus susceptibles d'être admis dans les salles d'urgence. Le revenu, le niveau d'éducation et aussi l'emplacement géographique sont des facteurs qui influencent I'utilisation de ce type de service.

\section{Problématiques émergentes}

«L e profil des F ranco0 ntariennes se rapproche davantage de celui des 0 ntariennes anglophones que des F ranco-0 ntariens »
Cette étude exploratoire qui trace un portrait de la santé des Franco- 0 ntariennes et des Franco- 0 ntarienslaisse planer une série d'interrogationstant du côté des stratégies de recherche que du côté des acteurs et actrices sociaux en cause. Tout d'abord, il importe de réitérer que l'approche épidémiologique comporte des limites, en particulier celle d'aborder l'ethnicité en tant que variable rattachée à un agrégat d'individus, masquant ainsi les rapports politiques entre groupesmajoritaires et minoritaires $\mathrm{D}$ ansle contexte d'une trèsforte politisation de la question de la santé, l'enjeu consiste à affiner les questions qui permettraient, lors de futurs sondages, de connaître davantage l'état de santé et de mieux-être des francophones.

Par contre, il importe de souligner que l'analyse de la trajectoire individuelle des francophones qui ont participé à l'Enquête sur la santé de l'O ntario de 1990 révèle d'importants clivages dans la distribution de la santé au sein même de la collectivité francophone. Les principales différences observées sont de genres et de générations. Elles font ressortir les éléments qui suivent.

Le profil des Franco- $O$ ntariennes se rapproche davantage de celui des 0 ntariennes anglophones que des Franco- $O$ ntariens. Elles se distinguent toutefois de ces premières en ce qui a trait au 
climat familial. Ce constat est problématique, compte tenu de l'importance qu'occupe la famille dans le maintien comme dans le rétablissement de la santé. À cet égard, ce rôle de la famille qui incombent largement aux femmes, s'accentue davantage en période de réduction du financement public.

La cohorte des hommes francophones âgés entre 45 et 64 ans s'est constamment démarquée au plan statistique comme étant plus à risque. $\mathrm{R}$ appelons que ceux-ci déclarent avoir plus de problèmes de santé et parallèlement, se perçoivent en moins bonne santé. Ils semblent prendre plus de risques et sont plus nombreux proportionnellement à consulter fréquemment un professionnel de la santé. $D$ e telles données invitent à étudier de plus près cette génération de francophones et leurs besoins. Les plus jeunes ne présentent pas de telles différences, sauf lorsqu'il s'agit du type de travail effectué par les personnes âgées entre 16 et 24 ans. Les adolescentes et adolescents de I'O ntario, en particulier les francophones, prennent-ils un mauvais départ en exerçant, dans une forte proportion, une routine professionnelle dangereuse?

L'enquête fait enfin ressortir que les aînés francophones, hommes et femmes, présentent un état de santé précaire. Francophones oubliés, il font figure de parents pauvres. Il est al ors essentiel de mieux connaitre leurs conditions de vie et leurs besoins d'autant plus que plusieurs aînés, et en particulier les femmes, vivent un état de pauvreté. Les statistiques révèlent que les aînés francophones ont un revenu considérablement inférieur à la médiane provinciale ${ }^{5}$. La fréquence d'accidents dont ils sont victimes requiert, de même, un examen de leur environnement immédiat en ce qui a trait à la question du logement.

«B énéficier d'un accès équitable aux services de santé est au centre des revendications de la population francoontarienne. »
Bénéficier d'un accèséquitable aux services de santé est au centre des revendications de la population franco-ontarienne. Les données nous manquent sur l'utilisation des services par les francophones et sur l'appréciation qu'ils en font. II importe de se tourner vers les études qual itatives afin de mieux appréhender cette question dans toute sa complexité. L'étude effectuée pour la Table féministe francophone de concertation provinciale de l'O ntario propose une réflexion dansce senset inviteàla recherche sur lesconditionsmenant à la prestation de meilleurs services sociaux et de santéé.

\section{Notes}


1. Vous pouvez communiquer vos commentaires à l'adresse suivante: Françoise B oudreau, Ph.D., D irectrice/ C hair, Département de Sociologie/ Sociology Department, Collège U niversitaire Glendon/C ollege, U niversitéYork/ U niversity, 2275 Bayview Ave, Toronto, O nt. M 4N 3M 6, Tél.: (416) 736-2100 \#88373 or 487-6741; Fax: (416) 487-6728; E-M ail: boudreau@erda.glendon.yorku.ca

2. R echerche réalisée sous l'égide de laTable féministe francophone de concertation provinciale de l'O ntario, en collaboration avec le R éseau des chercheures féministes de l'O ntario français, et grâce à l'appui financier des ministères ontariens des Services sociaux et de la Santé. N ous tenons à souligner la contribution particulière des personnes ayant participé, à différents titres, à I'équipe de recherche : D yane A dam, C aroline Andrew, Lyne B ouchard, Françoise B oudreau, Linda $C$ ardinal, D enyse C ulligan, $H$ élène D allaire, D iane Farmer, Gilles H uot, $M$ ichelle K érisit, D enise Lemire, $\mathrm{R}$ éjean $\mathrm{N}$ adeau et Anne $\mathrm{R}$ ochon Ford.

3. La variable francophone a été circonscrite à partir des questions suivantes du sondage : $Q$ uelle est votre identité ethnique ou culturelle? (française et canadienne-françai se) et Q uelle langue parlez-vous à la maison? (française et canadienne-française), ce qui est différent de la définition utilisée par Statistique $C$ anada.

4. Pour une définition de ce qu'on entend par routine professionnlle dangereuse, voir l'article de M cKellar dans le présent numéro.

5. O ffice desaffaires francophones, Les personnesâgées franco phonesen 0 ntario. Profil statistique, O ffice des affaires francophones, Toronto, 1996.

6. Voir l'article de B ouchard et C ardinal dansce numéro ou se référer à C arolineAndrew et al., Les conditions de possi bilité de services de santé et des services sociaux en français en 0 ntario : un enjeu pour les femmes, Table féministe francophone de concertation provinciale de l'O ntario, O ttawa, 1997. 